Article

\title{
Towards an Affordance-Based Ad-Hoc Suitability Network for Indoor Manufacturing Transportation Processes
}

\author{
Johannes Scholz ${ }^{1, *}$ (1) and Stefan Schabus ${ }^{2}$ \\ 1 Research Group Geoinformation, Institute of Geodesy, Graz University of Technology, A-8010 Graz, Austria \\ 2 Infineon Technologies Austria AG, A-9500 Villach, Austria; stefan.schabus@infineon.com \\ * Correspondence: johannes.scholz@tugraz.at
}

Received: 30 June 2017; Accepted: 31 August 2017; Published: 5 September 2017

\begin{abstract}
In manufacturing companies, productivity and efficiency are the main priorities, besides an emphasis on quality issues. The outcome of this research contributes to increasing production quality and efficiency in manufacturing. The article deals with indoor manufacturing environments and the transportation processes of production assets-referred to as smart transportation. The authors modelled the objects present in the indoor manufacturing environment with ontologies including their affordances and spatial suitability. To support flexible production and dynamic transportation processes have to be tailored towards the 'needs' of the production asset. Hence, the authors propose an approach utilizing an ad-hoc suitability network to support the "optimal" path computation for transportation processes. The objective is to generate a graph for routing purposes for each individual production asset, with respect to the affordances of the indoor space for each production asset, and measurements of a sensor network. The generation of the graph follows an ad-hoc strategy, in two ways. First, the indoor navigation graph is created exactly when a path needs to be found-when a production asset shall be transported to the next manufacturing step. Secondly, the transportation necessities of each production asset, as well as any disturbances present in the environment, are taken into account at the time of the path calculation. The novelty of this approach is that the development of the navigation graph-including the weights-is done with affordances, which are based on an ontology. To realize the approach, the authors developed a linked data approach based on manufacturing data and on an application ontology, linking the indoor manufacturing environment and a graph-based network. The linked data approach is finally implemented as a spatial graph database containing walkable corridors, production equipment, assets and a sensor network. The results show the optimal path for transportation processes with respect to affordances of the indoor manufacturing environments. An evaluation of the computational complexity shows that the affordance-based ad-hoc graphs are thinner and thus reduce the computational complexity of shortest path calculations. Hence, we conclude that an affordance-based approach can help to decrease computational efforts for calculating "optimal" paths for transportation purposes.
\end{abstract}

Keywords: indoor manufacturing; smart transportation; affordance-based navigation; linked manufacturing data; spatial graph database

\section{Introduction and Motivation}

The interest in indoor geography-related research is increasing, especially as humans spend almost $90 \%$ of their daily time inside buildings [1,2]. In fact, most manufacturing processes take place in indoor spaces. In this paper we focus on semiconductor manufacturing, that requires an indoor cleanroom environment for the manufacturing process chain. Currently, there are a number of initiatives subsumed under the umbrella of smart manufacturing or Industry 4.0 that strive to increase 
the efficiency of manufacturing processes. In this context, Geoinformation may contribute in terms of modeling indoor space [3] or decision support for manufacturing purposes [4-6].

The universe of discourse (UoD) under review in this paper is a complex and highly flexible manufacturing environment of a semiconductor company, described in detail by [3,4]. To illustrate the complexity in the manufacturing line, there are several hundred production steps necessary to manufacture a single microchip. Hence, the time to finish a product varies from several days to several weeks $[3,4]$. Furthermore, several hundred different products are manufactured at the same time in the cleanroom. The equipment is spatially distributed across the manufacturing site, as the production is not aligned on a conveyor belt. One single production operation may be executed on several equipment. In addition, several thousand production assets are present in the factory in different degrees of completion. The layout of the cleanroom changes frequently, due to maintenance tasks, dismounting and/or installation of manufacturing equipment.

Currently, humans mainly do the transportation of production assets. Operators load production assets on trolleys and deliver each item to the next manufacturing step-which is dependent on the product type. In order, to increase the efficiency of the manufacturing environment in terms of Industry 4.0, the overarching goal is the transportation of production asset by either an autonomous transportation or by an autonomous assistance of humans. Because each product class, present in the production line, has specific requirements on the chosen transportation route in conjunction with constant changes in the indoor layout, there is a need for a dynamic, context-sensitive transportation planning (see Figure 1).

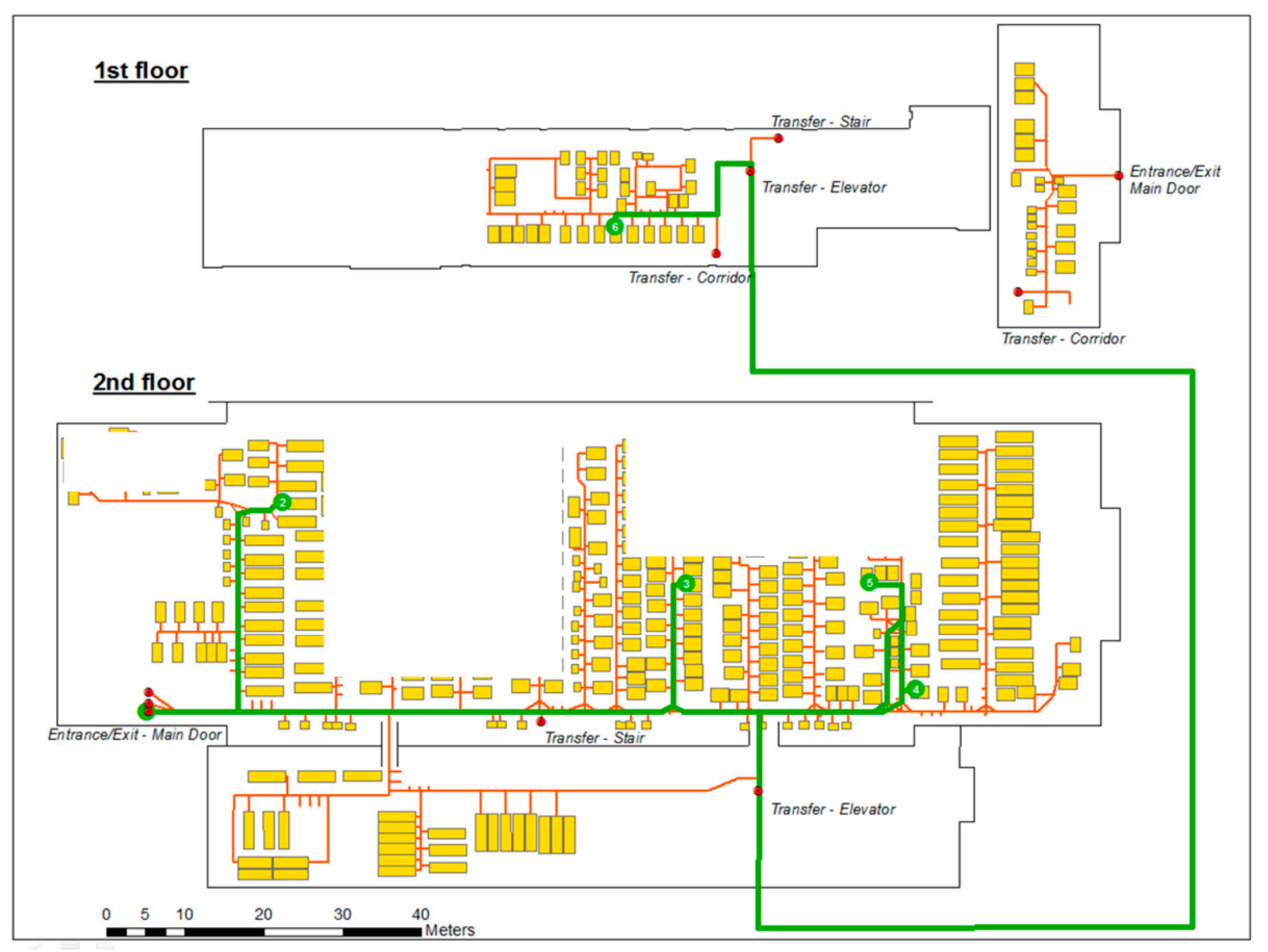

Figure 1. Prototypical application of an affordance-based path calculation for a given production asset (from [3]). The calculated path of a production asset, from entering the production line at node (1) and undergoing several manufacturing steps (nodes 2-6). 
In order to support context-sensitive transportation planning in an indoor environment an ontology is employed [3], that provides contextual information, linkages, and defined relations of the objects under review [7]. Based on the ontology, large volumes of spatial-temporal data originating from multiple data sources are combined, and analyzed to describe the production assets 'needs'.

Transportation planning and optimization in the context of this paper does not necessarily mean to minimize the physical transportation distance. Destinations-i.e., manufacturing equipment—and the path itself have to satisfy certain needs depending on the production asset at hand [8,9]. Based on the needs, we can calculate the suitability of possible transportation paths in the indoor environment. In this paper, we utilize the concept of affordances to calculate the spatial suitability-similar to that demonstrated by the authors of [3]. The term affordance is defined by $[10,11]$ as action possibilities perceived in a direct and immediate way. Additionally, the authors of [12] extended the theory of affordances to model spatial suitability. Furthermore, we follow an ad-hoc approach for the generation of the navigation graph. The ad-hoc aspect refers to the fact that a navigation graph is calculated for each production asset individually, when a path to the next manufacturing step is required. The calculation of the navigation graph and subsequently the "optimal" path adheres to the spatial suitability and relevant disturbances in the indoor environment at the time of the calculation-detected by sensor measurements. The combination of an affordance-based suitability network with an ad-hoc approach in an indoor environment has not been published before and extends previous papers [3,12-14].

The research question of this paper deals with the conceptual modeling of a context-sensitive, ad-hoc suitability network to support indoor manufacturing transportation processes. In detail, the paper strives to analyze if an affordance-based, ad-hoc approach to generate suitability networks for the calculation of "optimal" transportation paths in an indoor manufacturing environment reduces the computational complexity to calculate optimal paths-in comparison to the initial network. In addition, we analyze if the generated ad-hoc network allows re-routing if relevant incidents in the manufacturing environment occur. Generally, the ad-hoc aspect refers to the time of the network generation-i.e., after a manufacturing step has been finished-and the fact that each production assets' transportation requirements as well as quality relevant incidents have to be considered.

The structure of the paper is as follows: Section 2 starts with relevant work leading to the indoor manufacturing space and processes under review as well as a linked manufacturing data approach as spatial cyberinfrastructure comprising manufacturing data in Section 3. Section 4 defines the methodology for the affordance-based spatial suitability calculation focusing on ontologies, affordances and the suitability determination process and the ad-hoc aspect. Section 5 elaborates on the analysis of the proposed algorithm for the affordance-based ad-hoc suitability network to a classical shortest path algorithm. Section 6 highlights use-cases of the optimal path calculation in a manufacturing environment. Finally, a conclusion and a discussion is given in Section 7.

\section{Relevant Work and Research Approach}

This section highlights the literature related to this research and the general research approach followed in this paper. In the relevant work we elaborate on the contributions from GISc-in particular modeling indoor space, ontologies, and routing. In addition, Cyber-Physical Systems for manufacturing environments are of relevance for this paper. The description of the approach elaborates on the overall methodology followed in this paper.

\subsection{Relevant Work}

In order to support decision making in indoor manufacturing environments, there is a requirement to understand the manufacturing processes under review. A basic description of semiconductor manufacturing is given in [8]. Due to the emerging automation in manufacturing-known as smart manufacturing or Industry 4.0-there is a strong need to support decision making in order to enhance the competitiveness [15]. In the literature, there are several approaches to increase the efficiency 
of manufacturing lines $[6,15,16]$. To utilize optimization results [17] describe wearable devices for managers and employees of manufacturing companies. The authors of [4] propose that manufacturing decision making can be supported by adding the dimensions space and time to production related data.

Modeling indoor space and Geographic Information Systems for indoor purposes are active research fields, as there are a number of recent publications in this field. Modeling indoor space is of interest for GISc as a person resides approximately $90 \%$ of their daily time inside a building $[1,2]$. Modeling indoor space was first demonstrated by [18,19] focusing on wayfinding inside airports. In literature there are several approaches to model indoor spaces [20]. Ranging from topological models (e.g., [21]), hybrid models-where: both topology and geometry of the indoor space are part of the model [22-24], hierarchical models (e.g., [25]) and semantic models (e.g., [21,26]. In addition, Building Information models are used to model indoor space [27].

Ontologies, are an approach to formally describe a universe of discourse. A discussion of ontologies in scientific literature is given in [28,29]. An ontology according to [30] denotes the formal explicit specification of a shared conceptualization. There are different types of ontologies such as i.e., a domain ontology, representing a specific domain in an abstract way including the physical world and their behavior [19]. Authors in [31] adds entities, relations and rules as main elements to ontologies. Among others, references [32,33] elaborate on the spatial dimension of ontologies.

In order to support routing and navigation in an indoor environment several approaches exist in literature. A more general approach to model graphs for indoor routing and navigation is presented in [21]. The idea of graphs is based on the concept of duality [20,24]. In addition the OGC standard IndoorGML uses duality concepts for generating indoor routing graphs. The authors of [34] elaborate on an approach to compute indoor paths avoiding obstacles and groups of obstacles. In study [35], the authors propose a methodology to generate accessibility information for impaired people. The authors of [36-38] elaborate on the application of affordances for path calculation and decision making of pedestrian agents. In order to model spatial suitability, the authors of [39] provide a hierarchical representation of indoor spaces considering user groups and their tasks. Furthermore, the authors of $[12,13]$ provide an affordance-based approach to model spatial suitability for routing and navigation. In detail, they implement and evaluate the model on a routing scenario for mobility-impaired persons.

According to study [40], there is the need to integrate positioning data with domain-specific information to ensure data interoperability via linking data. The huge benefit of such linked geo-data is the improved data discovery and reusability of the data. Therefore, the authors of [40] developed a geo-ontology design pattern for semantic trajectories to show the applicability of linked geo-data on interdisciplinary, multi-thematic and multi-perspective data on the use-cases of personal travel and wildlife monitoring.

\subsection{Research Approach}

The overall approach followed in this paper can be described as follows. Based on an indoor ontology-describing the indoor manufacturing space-and spatial data on the indoor space, we evaluate an affordance-based routing approach for transportation tasks of production artefacts. The determination of a path in the indoor space is based on a network (i.e., a graph). The affordance-based approach, described in this paper, calculates individual suitability values (i.e., edge weights) depending on each production asset, and thus may reduce the complexity of the graph-which in turn reduces the computational complexity of a path calculation-e.g., shortest path. The novelty of this approach is, that this step is done with the help of an ontology.

In detail we develop a methodology to calculate individual spatial suitability values for the indoor space for each production asset at the time a route needs to be calculated. Additionally, this methodology supports the determination of the target of the route, based on the suitability values (i.e., if a certain manufacturing step can be performed on a piece of equipment). The result of this methodology is a network—called ad-hoc navigation network in this context—with calculated weights. 
The results are theoretically evaluated with respect to the computational complexity of a shortest path calculation. Finally, the approach is applied to a case study in a semiconductor manufacturing site.

\section{Indoor Manufacturing Space and Manufacturing Processes}

This section describes the indoor space and the objects in this environment, which is required to understand the necessity for an ad-hoc network generation in this context. The manufacturing context in this paper is a semiconductor production facility, which is special, due to the cleanroom conditions. This section relies on the work evident in studies $[8,9,17]$.

\subsection{Indoor Space of the Manufacturing Environment Under Rreview}

The indoor space under review is a semiconductor production facility. The manufacturing processes are done in a cleanroom environment. Any cleanroom ensures that the air inside has a low contamination with particles-in size and quantity. Cleanrooms are expensive to construct and maintain. Hence, they are constructed as compact as possible, which induces that the space for manufacturing equipment and movement of people and production assets is limited. In order to enter or leave a cleanroom there are defined entry points that are "secured" with airlocks. To change from one cleanroom into another requires the use of an airlock—in order to avoid the transfer of particles, especially when the cleanrooms are of different air quality classes. The cleanroom floor shows a special design that ensures a vertical laminar flow of clean air. The floor consists of single quadratic elements that reside on a frame structure-which might get bumpy due to the heavy wear or construction work.

In general, the movement of operators and production assets is restricted to the walkable areas of the cleanroom. Assets are transported on a trolley, which is pushed by a human operator or by an autonomous transportation via a transport system. Additionally, operators are allowed to carry production assets. The movement of operators and production assets might be restricted due to quality issues. Some asset types are prone to contamination from chemical processes. Hence, certain production asset types are not allowed to enter specific cleanroom areas-to avoid contamination. As the production facility is located on several floors, the production assets change between floors using elevators or staircases (requires the asset to be carried).

A spatially enabled sensor network is established in the manufacturing environment to ensure the quality of the cleanroom environment. The sensor network comprises of fix installed sensors with known locations measuring environmental parameters (i.e., air quality and contaminations) whereas moving sensors, which are located i.e., on trolleys, to detect bumpy floor areas. Generally, the sensor network supports a complete monitoring of each asset between and during manufacturing processes.

Manufacturing processes are a process chain that consist of a several hundred single steps-defined in a specific production plan. The production steps are not aligned on a conveyor belt, because the factory produces a high number of product types having different process chains. Additionally, each production step can be carried out on different equipment, which are geographically dispersed over the production facility. Thus, the transportation processes show a multifaceted structure due to the multitude of product types, according process chains/plans and manufacturing equipment. The degrees of freedom-due to the number of suitable manufacturing equipment for each manufacturing step and their geographical dispersion-present in the production line, indicate that transportation processes are a complex decision problem.

The production line differs from ordinary indoor spaces and production environments. Offices and/or residential buildings show a division into rooms and corridors. In a semiconductor manufacturing environment, rooms are hardly present, whereas corridors of considerable length are the main organizing structure. The layout of the indoor space under review is unstable, due to changing market demands. This requires equipment to be relocated, removed or the installation of new manufacturing equipment. The mentioned actions may change the layout of the production environment temporary or permanently, which has consequences for the transportation processes of production assets. 


\subsection{Spatial Cyberinfrastructure for Manufacturing Data}

The implemented spatial cyberinfrastructure based on a graph-database and RDF supports manufacturing data with near real-time capabilities-smart manufacturing. Due to the facts described in Section 3.1, the spatial cyberinfrastructure supports a semantic annotation-ontologies-including manufacturing data, tracked positions, historic processing information and future processing information. Therefore, a spatial graph database is implemented as basis for the spatial cyberinfrastructure-including the spatial and temporal dimension. This paves the way towards a just in time analysis and ad-hoc spatial suitability assessment to support navigation based on affordances in a proper manner.

Therefore, Figure 2 shows a visualization of the corresponding data described in Section 3.1 similarly to the Linked (Open) Data Cloud [41]. Figure 1 subsumes ontology classes for semantic annotations, historic and future manufacturing data and spatial information. The example shows an abstract basic top-level of the Linked Manufacturing Data, in which the physical location unions semantic annotations in blue, spatial information in green and manufacturing information in yellow. Therefore, Schabus, S., et al. [42] give examples how linked manufacturing data supports historic and future processing data.

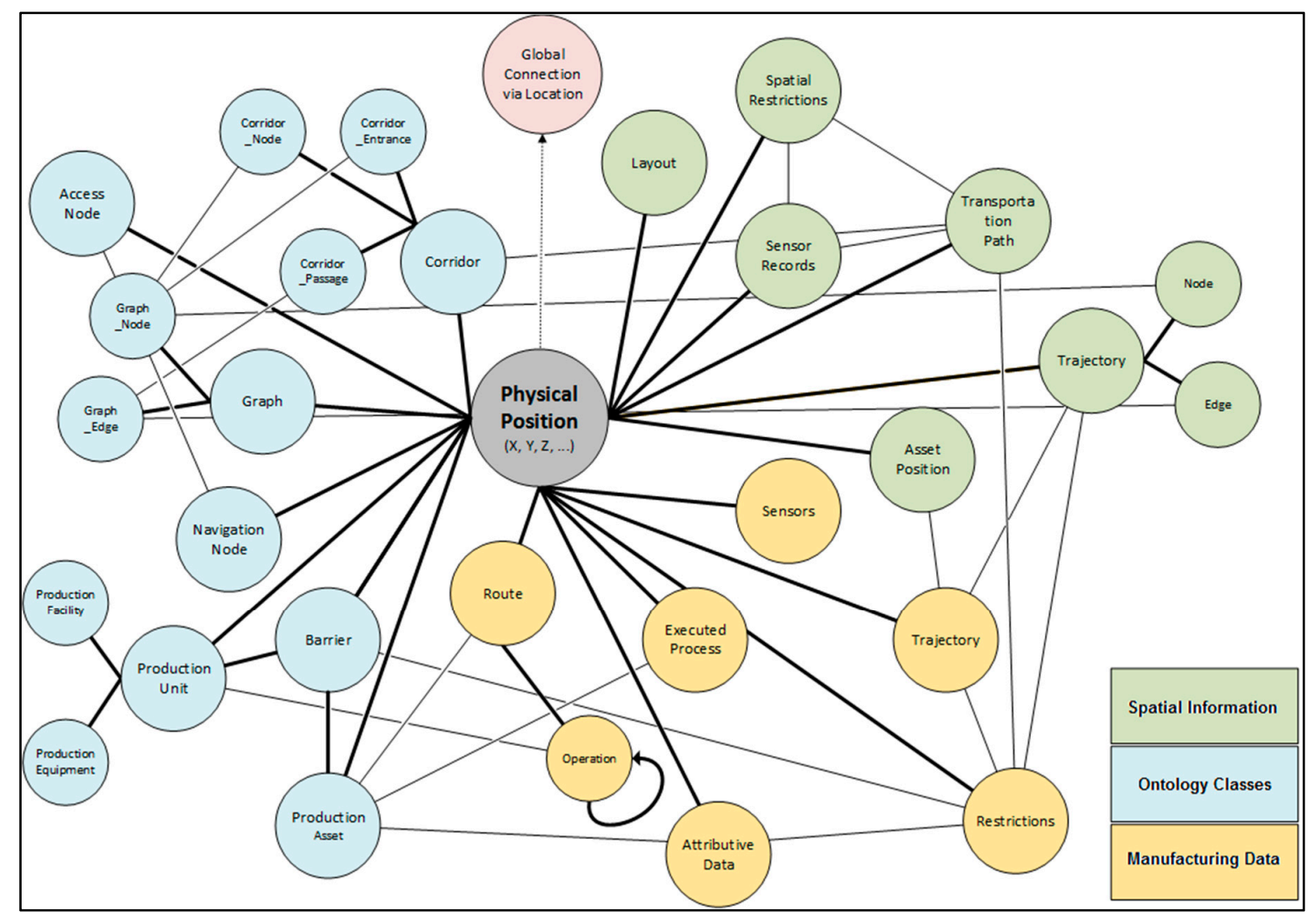

Figure 2. Linked Manufacturing Data for Transportation Tasks according to studies [3,41] at an abstract top level. Thick styled links represent the hierarchical structure of linked data, whereas thin styled links represent data links between different data sources.

\section{Methodology for Affordance-Based Spatial Suitability Calculation}

In the semiconductor manufacturing environment the production assets are transported from one manufacturing equipment to the next processing step. Each production asset (type) has peculiarities that have to be considered when planning a certain transportation route. The contemporary workflow in the manufacturing environment requires humans to decide on a path. Due to the presence 
of several hundred different production equipments, an operator can hardly know all relevant asset characteristics.

\subsection{Ontologies and Affordances}

The approach proposed in this paper is based on the concept of affordances [10,11], and is similar to that presented in [12]. The term affordance, coined by Gibson [10,11], based on the verb "to afford" is defined as follows:

"The affordances of the environment are what it offers the animal, what it provides or furnishes, whether for good or ill" ([11], p. 127).

Thus, a chair offers the possibility of seating for a human being. The offering "to sit" is a result of its properties, and the capabilities and properties of the acting agent as well [11]. Hence, a chair designed for humans, does not afford sitting for an elephant, due to the size and weight of the animal in relation to the properties of a chair. Hence, with respect to Koffka [43], the term affordance can be expressed by the following sentence: "Each thing says what it is" ([43], p. 7).

In the field of GIScience, Jordan et al. [44] utilized affordances for modeling places in a GIS. They propose to model three aspects in order to describe a place: agent, environment and the task. In [44] the affordances of a restaurant are mentioned as example, where the authors evaluate the suitability of restaurants for customers. Therefore, the capabilities and preferences of the agent (i.e., customer) and the task (e.g., socializing, eating) need to be defined.

In the specific context, the determination of affordances of each indoor entity was done in a semi-automated way. First, the objects were analyzed regarding their connectivity (e.g., different floor levels, connecting different halls) and their navigation "offerings" (e.g., turn right, left). In addition, the offerings in terms of manufacturing capabilities and restrictions were determined by analyzing manufacturing related data.

\subsection{Determination of Spatial Suitability}

In the context of this paper-production assets residing in an indoor manufacturing environment-several production asset types are present. Each production asset type shows specific properties that have to be respected. In order to decide on a transportation path for a production asset, a destination point and a path connecting destination and current position with its suitability, need to be determined. In this process, finding a destination point equals to finding a manufacturing equipment offering a certain production process.

The methodology relies on characteristics of each production asset that are as follows:

- Product type: The product type provides implicit information on the manipulation of the production assets. Specific types need to be handled with care, as they might break easily. Thus, transportation over stairs or "bumpy" cleanroom sections are restricted. Other types are able to move through contaminated or low quality cleanroom areas due to a specific enclosure. The mentioned enclosure has to be carried with both hands, which means that the operator is not able to open doors. In addition, the product type defines other impediments to transport, such as air quality or contamination risks.

- List of manufacturing operations: This information stores the sequence of manufacturing processes that have to be carried out. As several processes can be performed on several pieces of equipment, the resulting quality of the manufacturing processes may differ. Hence, each production asset should choose a production equipment that fits 'best' in terms of manufacturing quality.

To calculate spatial suitability, we break the processes down into tasks and sub-tasks-which follows the approaches of the authors of [12] as well as [45,46]. The methodology-depicted in Figure 3-decomposes each transportation task for each production asset, starting from the overarching 
next objective, e.g., "move to the next production step 'ion implantation' starting from cleaning station \#5". The algorithm identifies the sub-actions of this intended transportation process, based on the following procedure: The algorithm analyzes available equipment offering "ion implantation" with respect to the production asset at hand. If there is more than one equipment offering the manufacturing step, the algorithm looks at additional properties with respect to the production asset-e.g., defect and/or failure rates-in order to apply a weighting of the equipment. Each target equipment, that affords the manufacturing process, is analyzed in comparison to the source equipment-here the source is the cleaning station $\# 5$ and the target is an 'ion implantation' equipment. This results in geographical differences-e.g., different floors, different location in the production hall. In order to determine the sub-actions, the algorithm then "moves" backwards from the target to the source-i.e., tries to reach the source. In this way, the sub-actions can be determined-like 'switch floor, 'change production hall'.

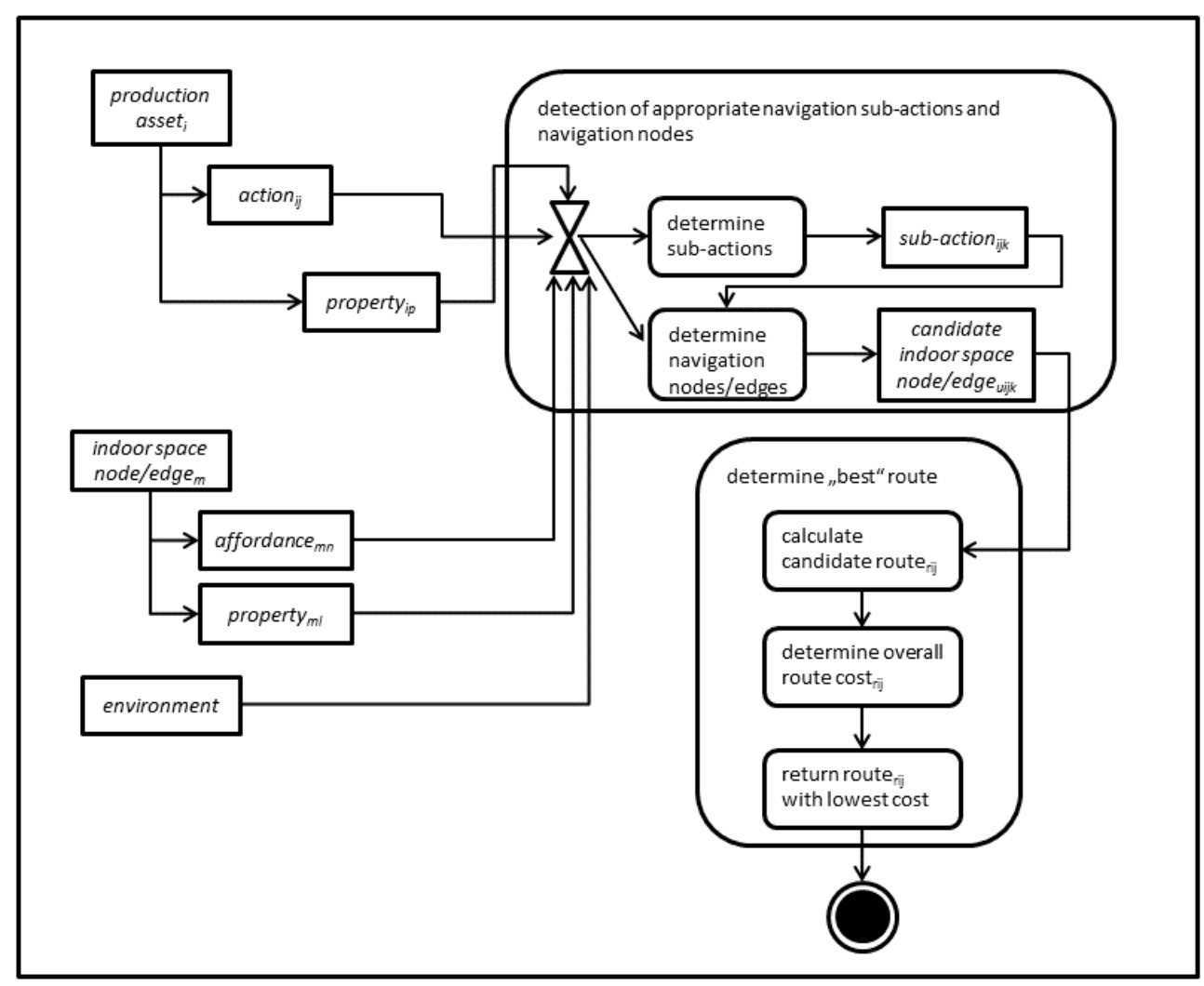

Figure 3. Approach to calculate the suitability of possible routes in an indoor environment, based on affordances (from [3]).

Based on identified sub-actions, the algorithm determines locations and indoor entities that afford the necessary sub-actions. An example for an indoor entity that offers a sub-action called, 'transfer from floor 3 to floor 2', is an elevator. Similar to the approach in [14], we state that the affordances are not strict binary properties. We propose that affordances should be modeled close to the concept of suitability. Hence, in this paper we express an affordance as a rationale number with respect to the environment, the task and the agent. For instance, a staircase and an elevator afford to change the floor level in a semiconductor manufacturing environment. Nevertheless, most operators would prefer the elevator, due to the reduced risk of falling and damaging the assets. The suitability is expressed in the following formulation.

A production asset-denoted as agent $t_{i}$ has several actions to perform-denoted as action ${ }_{i j}$. Actions are specific for each agent, which justifies the indexation $i, j$. Each agent has a set of properties, where each property is indexed with $p$. Hence, the properties for each agent are stored in the variable 
property $y_{i p}$. A traversable network consisting of vertices and edges, where nodes have associated properties and affordances, represent the indoor environment. Each network node ${ }_{m}$ has multiple affordances stored in variable af fordance fn $_{\text {, }}$ and several properties denoted as property $y_{m l}$. Multiple affordances and properties are necessary to describe different actions/tasks possible at each node.

The algorithm determines sub-actions for each asset, which is a gradual refinement of action $i j$. Each action is broken down into sub-actions until each sub-action is decomposed to the basic level. The basic level is reached when each sub-action can be matched with an affordance of a node-i.e., when a node can fulfill the action needed. An example is the movement of a production asset from equipment $X$ to another equipment to perform operation $Y$. First, the algorithm determines which nodes-i.e., equipment-are offering operation Y. Assuming that the equipment offering operation $Y$ is on another floor, the algorithm identifies sub-actions like 'change floor'. Subsequently, the algorithm determines navigation nodes that fulfill the need 'change floor'-i.e., searches for nodes affording changing the floor.

In addition, the property $y_{i p}$ of production asset ${ }_{i}$ needs to be considered in determining the suitability of a specific node or edge. This represents the suitability of a navigation node or edge to be traversed by $a_{s s e t}$. The suitability of a navigation node or edge for $s_{u b}$-action $_{i j k}$ is a function of the affordances and properties of indoor space node ${ }_{m}$ or indoor space edge $e_{m}$ and the sub-actions and properties of $a_{s s e t}$. $_{i}$ An example is the suitability of a staircase or a bumpy ground to be traversed by a thin product asset. In both cases, the properties of the indoor nodes in conjunction with the properties of the thin asset, result in a low suitability. This is due to the fact, that this thin production asset can only withstand low vibrations and shocks. As a result we get the list of indoor space nodes and indoor space edges with their according suitability values for asset $_{i}$.

The suitability values are defined as the quotient between properties and capabilities of $a_{s s e t}$ and the corresponding offering of the indoor node or edge for ratio scaled properties, like acceleration values, particle concentration per $\mathrm{m}^{3}$ air, or manufacturing defect rate of the asset type at a specific equipment. For binary values, like accessibility (true/false) we use a binary suitability value. The suitability values present at a specific edge and node are added up in order to get one suitability value per graph element. If one suitability value equals to zero, we regard the edge or node as not traversable.

The resulting lists of candidate indoor space nodes $s_{u i j k}$ and candidate indoor space edges uijk $_{\text {serve as }}$ basis for the generation of the candidate routes ${ }_{r i j}$ (for each action $_{i j}$ ). A candidate route is defined as a traversable connection between two indoor navigation nodes-that represent the beginning and end of a task or an action. An example is the movement from equipment $X$ to an equipment offering the next manufacturing process $Y$. As several equipment may exist that are capable of performing operation $Y$, and several possible paths connecting equipment $X$ and the equipment offering operation $Y$, the algorithm may end up in suggesting several different candidate routes. By using a shortest path algorithm-Dijkstra - with (a) length (distance) and (b) suitability values as costs we are able to compute the most suitable route with respect to the action of the specific production asset.

\subsection{The Ad-Hoc Aspect}

The calculation of an optimal path for each production shall avoid potentially harmful spots in the manufacturing space and shall react dynamically on equipment breakdown or existing bottlenecks. Hence, the calculation of an "optimal" path containing all manufacturing steps-from raw to final product-seems not advisable, as the conditions in the manufacturing environment may change quite rapidly. This is based on e.g., equipment breakdown, relocation, or removal. Additionally, the constant change of market demand and altered production necessities may require a shift in the production capacity. Additionally, incidents-like contamination issues, or malfunctioning airlocks-may happen on a random basis, which need to be considered when generating an optimal path.

In order to overcome the aforementioned issues, we propose generate the spatial suitability graph and the path calculation in an ad-hoc manner. This ad-hoc aspect is realized by a calculation of the 
individual suitability network immediately after a production asset finishes a manufacturing step. At this stage, a production asset requires to be transported to the equipment capable of performing the next manufacturing step. Exactly at this stage the algorithm is able to consider the "state" of the manufacturing environment in relation to the specific asset-e.g., contamination, bumpy floor. If the indoor space might be harmful for the quality of the specific asset, then the corresponding edge and/or node is not included in the suitability network.

An example for such an incident is a sensor that monitors particle contamination in the indoor cleanroom. Let us consider that the air cleaning system in a particular area is malfunctioning, resulting in a high concentration of particles in the cleanroom. In the manufacturing site under review the wafers are stored in different types of boxes. Box Type 1 can be opened any time by humans, whereas box type 2 keeps wafers in a secured and controlled environment, sealed off from the environment, and cannot be opened by humans-only by specific manufacturing equipment. Hence, boxes of type 2 are not endangered when being transported through an area with a malfunctioning air cleaning-whereas boxes of type 1 can be contaminated with particles and damaged.

According to the Merriam-Webster dictionary the term ad-hoc is defined as being for a "particular end or case at hand without consideration of wider application". As the suitability network generated for each production asset, at each time it completes a manufacturing step meets the aforementioned definition-of being calculated for one specific case without wider application-we regard the suitability network having an ad-hoc character. This can be further justified, as the suitability network might look different for each individual asset, and looks different for similar assets depending on their specific position in the manufacturing environment and or degree of completion.

In order to address such ad-hoc aspects we utilize a spatially enabled sensor network distributed over the indoor space. The sensors detect possible contamination risks, as they measure air quality (particles density) and gas concentration throughout the manufacturing facility including their own position. In addition, accelerometers mounted on the production equipment and transportation carts detect uneven surfaces. The sensor measurements are stored in the data storage in near-real time and thus can be utilized when calculating the suitability values.

Hence, the approach is able to rely on the current state of the cleanroom environment-which is reflected in the suitability values. This ensures that the algorithm reacts to immediate disturbances, problems in the manufacturing facility, by an ad-hoc generation of the navigation network each time the calculation is done. At each calculation the approach establishes the traversable graph-consisting of nodes and edges), and determines the suitability values of each node/edge.

\section{Analysis of the Affordance-Based Spatial Suitability Network for Shortest Path Calculation}

The developed approach of the ad-hoc suitability network described in Section 4 is compared with the Dijkstra shortest path algorithm. Therefore, we compare the size of the graph-edges and nodes/vertices-and the computational complexity of the algorithm. Then, we evaluate the computational complexity based on an example, comparing the complete graph or the graph induced by the affordance based methodology. Next, a single 'personalized' transportation process for one production asset in a small area of a production hall is highlighted. This small area is then used for the assumption of the computational complexity of one complete production hall. Due to confidentiality reasons, we are not allowed to use accurate values for distances. Additionally, we have to disguise the manufacturing layout due to confidentiality.

In Table 1 we compare the size of the graph. First, we analyze the complete graph, which is a basis for the Dijkstra shortest path algorithm-denoted as $G(V, E)$. For the ad-hoc network we use $G_{A H}\left(V_{A H}, E_{A H}\right)$. The ad-hoc network graph is a subset of the complete graph $G_{A H} \subset G$, because edges and nodes that do not afford the 'needs' are excluded. Hence, the computational complexity of the Dijkstra algorithm $O=(|V| * \log (|V|)+|E|)$, is higher than the computational complexity of the ad-hoc network $O_{A H}=\left(\left|V_{A H}\right| * \log \left(\left|V_{A H}\right|\right)+\left|E_{A H}\right|\right)$, if the same graph is the overall basis of the computation. 
Table 1. Comparison of a classical Dijkstra shortest path algorithm and the Ad-Hoc Suitability Network Algorithm to identify optimal indoor transportation.

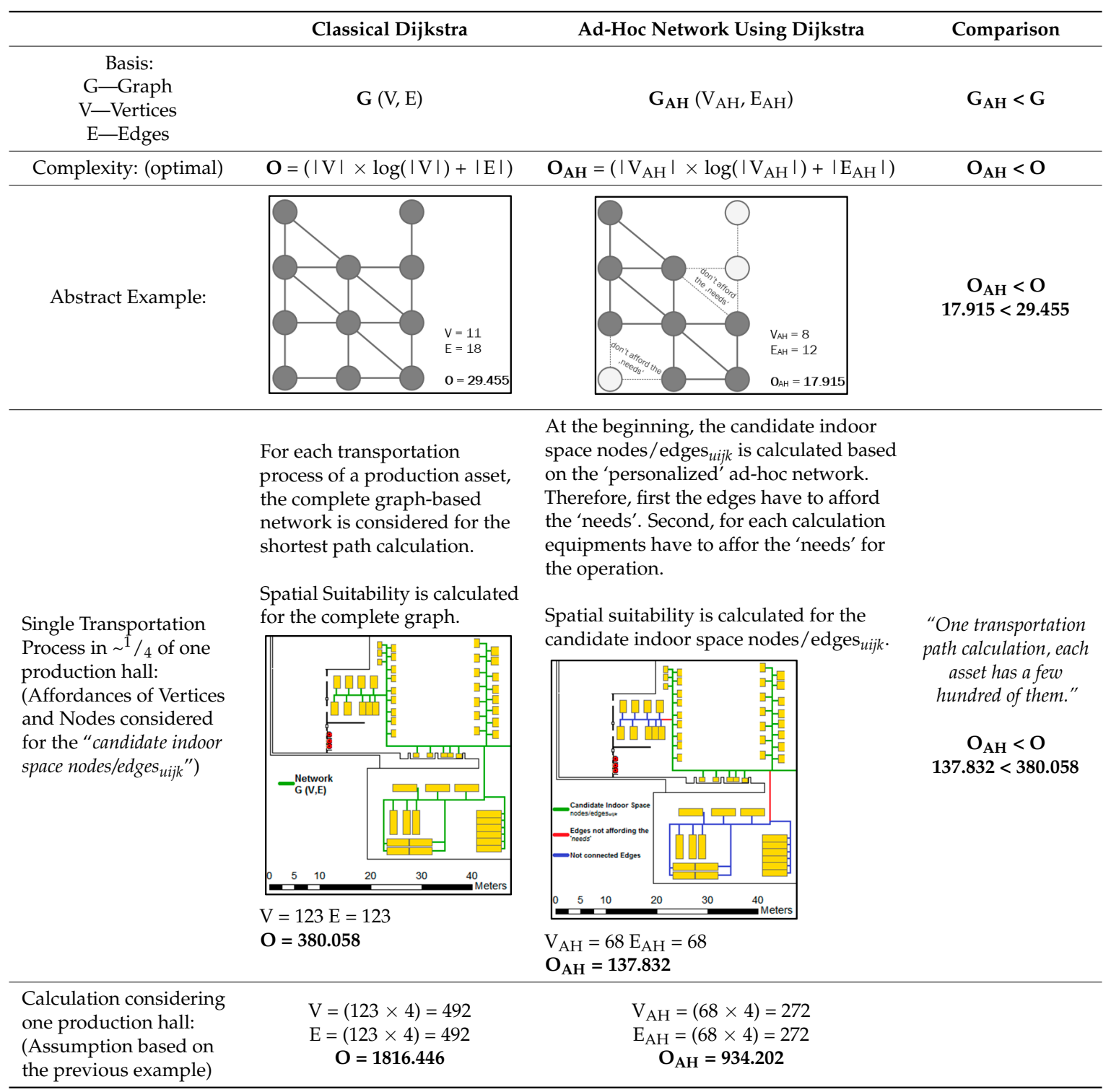

Note: Exact values are prohibited and white spaces are involved to disguise the layout of the production environment.

A practical example focuses on the transportation process of a single production asset in a small area of a production hall. The approach using only a shortest path algorithm considers the complete existing graph, which is marked with a green line. The ad-hoc suitability network defines the candidate indoor space nodes/edges ${ }_{u i j k}$-already excluding edges and nodes that do not afford the 'needs' of the specific production asset-before calculating the shortest path. This reduced graph is used for determining possible routes ${ }_{\text {rij }}$ subsequently. These routes $_{\text {rij }}$ are then used for the spatial suitability assessment. Here, the number of possible routes is less than for the complete graph.

\section{Case Study: Optimal Path Calculation in a Manufacturing Environment}

This section describes a case study of the implemented affordance based calculation of optimal paths in the manufacturing environment based on an ad-hoc suitability network. Thus, a proof of concept of the implementation is provided in Section 6.1 of the determination of the ad-hoc suitability network. Section 6.2 addresses the decision of the optimal route based on defined affordances and shows how the optimal can equal the shortest path and how it can differ. 


\subsection{Implementation of the Ad-Hoc Suitability Network for a Smart Transportation Process in a Flexible Manufacturing Environment}

The implementation of the workflow presented in Section 4.2 results in a developed Java Application. This application utilizes a previously developed spatial graph database, presented as linked manufacturing data approach [42]. The linked manufacturing data approach serves as basis for the research as it provides the digital representation of the indoor manufacturing environment at hand, the corresponding manufacturing data and semantic annotations of manufacturing data and the environment. Therefore, the spatial graph database is queried from the implemented Java Application via implemented procedures in Cypher and comparison of semantics and manufacturing data is done in the computed application.

Intermediate results of the implemented approach can be seen in Figure 4. In Figure 4, the first intermediate result is depicted as the basis network for the overall route calculation, layout and equipment as well as the starting point for the transportation process. The next two intermediate results in the middle of Figure 4, focus on the affordances of the nodes or edges derived by the linked manufacturing data approach and the semantics. A comparison is made separately if the edges/nodes afford the needs of 'to transport'. On top, edges are matched with their affordances and if they are suitable for the production assets' needs or not. Green edges afford the needs and the red edges do not afford the needs. Blue edges afford the needs, but are not connected anymore. The compared nodes for simple action such as turn left or right afford the needs, except nodes representing an equipment. These are matched with the next operation and if the equipment support the needs for this operation. Such suitable equipment is visualized in green. The combination of both intermediate results presents the candidate indoor space node/edge $e_{\text {uijk }}$ as intermediate result and as basis for the further suitability assessment for each possible route. Figure 4 shows as a result the 'personalized' ad-hoc network for a production assets' transportation task, as combination of affording nodes and edges. The result shows that only suitable equipment is connected to the network if there is a possible connection via edges. A topology check removes edges with only one node (blue lines).

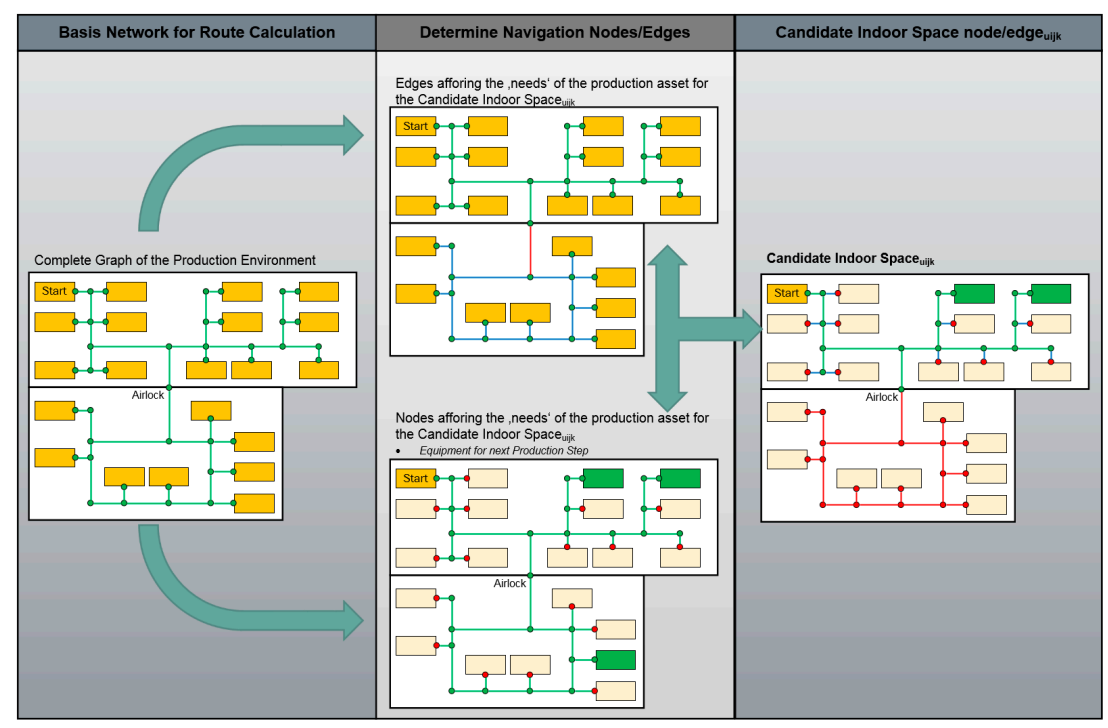

Figure 4. Intermediate results of the implemented approach from the complete graph as starting point

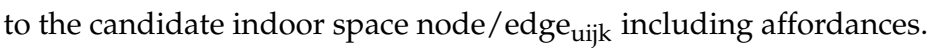

The candidate indoor space node/edge $e_{u i j k}$ is then refined by the developed application, as edges and nodes which are not part of a possible route to suitable equipment are removed. Therefore, built-in shortest path queries are used from neo4j spatial queried by the application. For this calculation, a default length of zero is assumed to return all possible paths from the starting point to each suitable 
equipment. Figure 5 shows further results of this calculation of candidate routes, with the basis of the candidate indoor space uijk with affording edges and nodes (in green) on the left side. In the middle, Figure 5 depicts the next limitation of the ad-hoc network comprising only the candidate routes $_{r i j}$. On the right side each route is displayed separately.

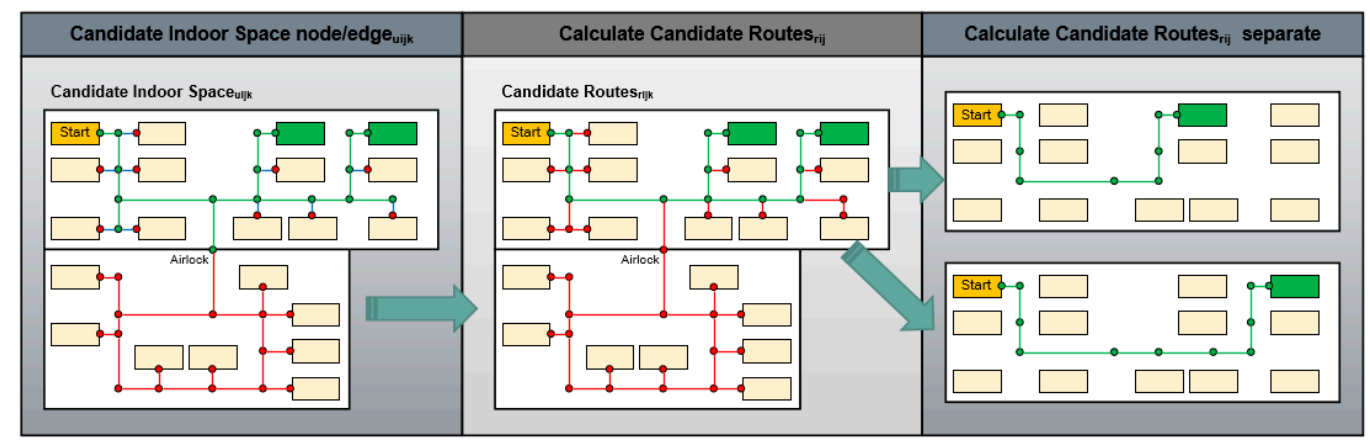

Figure 5. Intermediate results showing the limitation of the candidate indoor space node/edge $e_{u i j k}$ to all possible candidate routes $_{\text {rij }}$.

This candidate routes $r i j$ are the basis for the suitability assessment, and thus the final result of the ad-hoc suitability network. Therefore, the sensor network is mapped to the prospective candidate routes via either a spatial join of the sensors and nearby edges or via a fixed definition of the sensor and possible affected areas/edges. This suitability assessment of the sensor measurements can be triggered via events, whereas only the candidate routes have to be adjusted and re-calculated for the final route decision or a detour. The sensor measurements are weighted according to the production assets' affordances and mapped onto the corresponding edge, which is then used to identify the route with the least cost as the optimal path.

In comparison to existing approaches, the presented methodology outlines the optimal path computation based on an ontology. The proof of concept utilizes affordances of the indoor production environment and the production asset. The optimal path computation considers what the indoor environment offers to the production asset and identifies the individual transport suitability alongside with disturbances occurring in the indoor space. Hence, our approach has the advantage of reducing the computational complexity of path calculations, due to a reduced navigation network. In comparison, authors of [47] focused on a semantic navigation approach focusing on human navigation. They developed an approach to calculate the best traversable path between a start and an end point, with respect to the user's capabilities. Nevertheless, the paper does not utilize the concept of affordances explicitly. In addition, the paper does not include an ad-hoc component, as users are redirected to the original planned path, in case they get lost-neglecting the possibility of generating a new path. In the studies [48,49] the authors present empirical studies identifying the least risk path for human indoor navigation. In the papers a path between two points are calculated that has the least risk of getting lost. Thus, the papers highlight an approach for calculating a least-risk path without including different user's preferences. The research in this paper focuses on generating optimal paths for production assets, which might have a certain risk of getting lost. However, the production asset is the focal point for the path computation and not the human, as will be equipped with an indoor navigation assistance system.

\subsection{Use-Case: Optimal Route Decision Based on Affordances and the Ad-Hoc Suitability Network}

The identification process of the optimal route is based on the suitability assessment of the

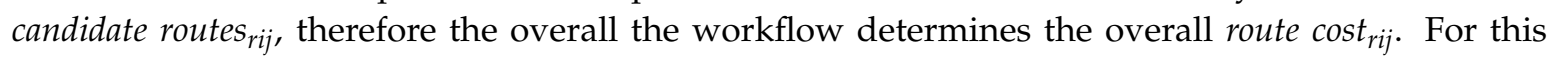
suitability assessment, the sensor measurement values are compared with affordance. The developed application classifies the measurement value, the worse the measurement value, the higher is the 
impact factor of the sensor on nearby affected edges which is multiplied with the length of the edges. Finally, the application returns the route ${ }_{r i j}$ with the lowest cost representing the optimal path in terms of length and quality. This is applied on two case studies based on the before created ad-hoc suitability network and the corresponding candidate routes ${ }_{r i j}$.

The first case study is depicted in Figure 6 with the suitability assessment of the two routes named Route_X1 and Route_Y1. One sensor (highlighted with a bigger size) triggers an event as a threshold is exceeded, which is considered for the identification process of the optimal path. As it can be seen in Figure 6, this alerting sensor is affecting Route_X1 and Route_Y1. Therefore, for each route the determination of the overall route cost is done by summing up the respective edge lengths. Thus, in Figure 6 the length for each edge is stated and the thickness of the edge shows, if the edge is affected or not. The impact factor of the measured value representing the suitability is defined as two for sensor1. The calculation of the shortest path is with $30.2 \mathrm{~m}$ for Route_X1 and $39.2 \mathrm{~m}$ for Route_Y1. By calculating the optimal path and thus by incorporating the suitability assessment based on affordances, Route_X1 results in a value of 39.4 and Route_Y1 in a value of 49.1 as the affected edge is multiplied with the impact factor. This case study shows, that both routes are affected similarly by the sensor measurement and the optimal path equals the shortest path.

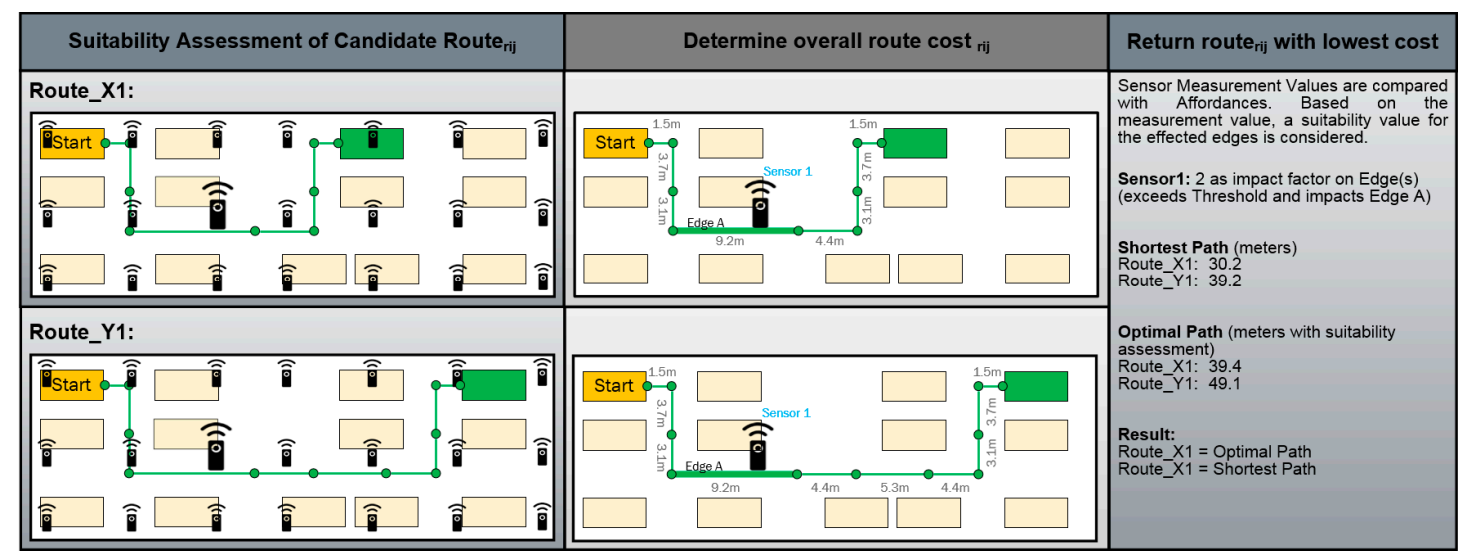

Figure 6. Use-Case: Route determination based on the ad-hoc suitability network where the optimal path equals the shortest path.

The second use-case focuses on the difference between the optimal path and the shortest path. Therefore, Figure 7 has the same basis as Figure 6 with two routes named Route_X2 and Route_Y2, whereas two different sensors Sensor 2 and Sensor3 exceed the defined thresholds. Based on the spatial distribution of the sensors, Sensor2 affects Route_X2 and Route_Y2 contrary to Sensor 3 affecting only Route_X2. The impact factor of Sensor2 is determined as 2 and the impact factor of Sensor 3 as 3, as a worse value was measured. The calculation of the classical shortest path equals the calculation of the first use-case. The difference is in the calculation of the optimal path combining suitability and length. Route_X2 has more edges which are affected by the sensors than Route_Y2, also with a stronger impact factor. Therefore, the calculation of the optimal path shows that Route_X2 has a value of 45.1 and Route_Y2 a value of 44.3. This means, that Route_Y2 is the optimal path and that the optimal path does not equal the classical shortest path depicted in Figure 6.

The case studies show that a longer path is accepted if it is necessary to maintain the quality of the production asset, or if the subsequent manufacturing step is not reachable on the shortest path-e.g., due to the inherent risk for the production asset. Hence, it seems advisable to make a detour to avoid e.g., areas with a bumpy floor with a thin wafer, as they might break easily. A damaged or contaminated production asset-i.e., a silicon wafer-has to be discarded. Depending on the degree of completion of the asset the company loses the invested manufacturing capacity and time (several 
hours to several days/weeks). Thus, any manufacturing company is committed to avoiding quality risks and to reducing scrap.

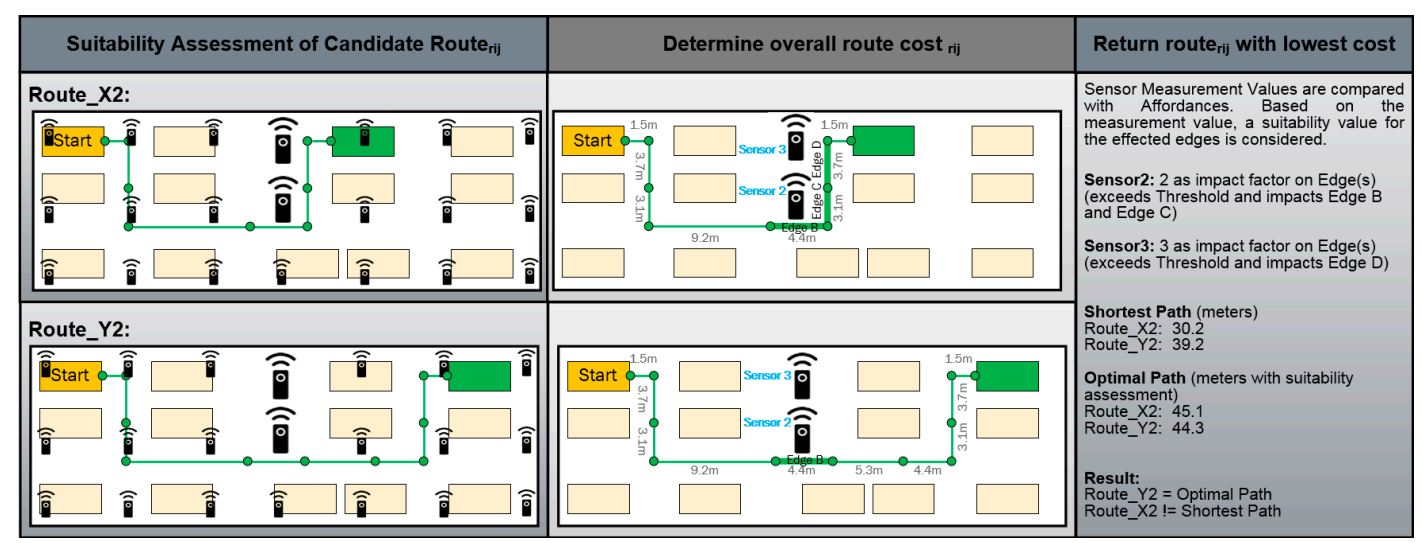

Figure 7. Case study: Route determination based on the ad-hoc suitability network where the optimal path does not equal the shortest path.

\section{Conclusions and Discussion}

To sum up, the research elaborates on the development of an ad-hoc suitability network for indoor manufacturing based on a previously developed linked manufacturing data approach [42] and an indoor ontology for indoor manufacturing environments [3]. The calculation of an ad-hoc suitability network utilizes the context of production entities, affordances of manufacturing environments, and a sensor network to calculate the spatial suitability (for each individual asset). Based on the spatial suitability the approach calculates the optimal path to transport assets along a graph-based network. The path starts at the last processing equipment and the possibility of multiple target equipment if they afford the 'needs' of the asset. The ad-hoc suitability network is the basis for identifying the optimal path. In the best case, the optimal equals the shortest path if no sensor measurements exceed a defined threshold. Otherwise, the optimal path can be a detour to avoid potential contamination or other detected incidents. A proof of concept is implemented showing the variability of the ad-hoc suitability network if sensor measurements change. This smart transportation approach uses a spatial graph-database to store the linked manufacturing data, which can be considered as cyber-physical system.

The ad-hoc strategy of the approach is found in two different aspects. First, the suitability network is generated in an ad-hoc manner-exactly when a production asset finishes a manufacturing step and an optimal transport path to the next production step is required. Hence, we calculate a suitability network and an optimal path for each individual production asset. Secondly, the generation of the suitability network considers current disturbances and incidents in the indoor environment, such as contamination issues, air quality problems. Hence, the approach is intended to avoid such areas which are harmful for the quality of the production assets. In this paper, we utilize a sensor network that observes the manufacturing space for any disturbances.

We highlighted the benefit of semantic annotated manufacturing data for the creation of an affordance-based ad-hoc network including only those edges and nodes that afford the 'needs' of the respective production asset. An additional benefit is the use of semantics for the spatial suitability assessment, to link the sensor network with transportation processes and the solving of interoperability issues in the production environment. The novelty of this approach is given due to the fact, that the graph simplification process is purely based on the suitability calculation-which itself is based on an ontology. Thus, we see the combination of an ontology, reasoner, and (shortest) path calculation as a novel idea. The approach is justified by the fact that the manufacturing space under review is highly flexible with several hundred production assets in the facility at the same time, having different 
degrees of completion. It would be quite difficult to model the interdependencies between indoor space, production assets (at different completion degrees) in a standard database or mathematical model. In our opinion only ontologies/semantics can handle this complexity at hand.

The approach helps to generate a thin navigation network, which reduces the computational complexity of shortest path calculations. Thus, shortest path calculations, necessary for each individual production asset when completing a manufacturing step, require less computing power compared to using the full navigation network.

In terms of the research question, a context-sensitive approach is developed that calculated an ad-hoc suitability network to support indoor manufacturing transportation processes. The results show that our ad-hoc suitability network generates thinner navigation networks, which in turn reduces the computational complexity of shortest path algorithms. As the calculation of transportation paths is a very frequent task, this approach reduces computational power necessary.

The ad-hoc aspect is shown in the case study, where a sensor network monitors the manufacturing environment. If an incident results in a sensor measurement that exceeds the production asset's individual threshold, then the edge or node is not included in the suitability network. Hence, the asset avoids harmful areas.

Future research includes the application of the approach in autonomous transportation solutions in manufacturing environments. Here transport robots are able to load and unload production assets and to move them to the next production step in an autonomous manner. Such a solution needs to consider the spatial suitability for the production asset and the transport robot itself in order to succeed. Before the autonomous transportation solution is implemented, humans pushing trolleys to transport production assets to the next manufacturing step could be supported with an application following a location-based service approach. Therefore, operators transporting assets are supported in their route decision (i.e., they get a recommendation), and are alerted when they should load/unload a specific asset. Such a solution requires some kind of optimization in order to find the "optimal" route for a number of assets. Hence, coupling the ontology —and affordances-with a mathematical optimization approach seems to be a promising approach to solve this issue.

Acknowledgments: Supported by TU Graz Open Access Publishing Fund.

Author Contributions: Johannes Scholz and Stefan Schaubs developed the algorithmic approach. Johannes Scholz wrote Sections 1 and 4. Stefan Schabus implemented and conducted the experiments and wrote Sections 5 and 6. Johannes Scholz and Stefan Schabus wrote Section 2, Section 3, Section 7 together.

Conflicts of Interest: The authors declare no conflict of interest.

\section{References}

1. Jenkins, P.L.; Phillips, T.J.; Mulberg, E.J.; Hui, S.P. Activity patterns of Californians: Use of and proximity to indoor pollutant sources. Atmos. Environ. Part A Gen. Top. 1992, 26, 2141-2148. [CrossRef]

2. Klepeis, N.E.; Nelson, W.C.; Ott, W.R.; Robinson, J.P.; Tsang, A.M.; Switzer, P.; Behar, J.V.; Hern, S.C.; Engelmann, W.H. The National Human Activity Pattern Survey (NHAPS): A resource for assessing exposure to environmental pollutants. J. Expo. Anal. Environ. Epidemiol. 2001, 11, 231-252. [CrossRef] [PubMed]

3. Scholz, J.; Schabus, S. An indoor navigation ontology for production assets in a production environment. In Proceedings of the 8th International Conference on Geographic Information Science, GIScience 2014, Vienna, Austria, 24-26 September 2014; Duckham, M., Pebesma, E., Stewart, K., Frank, A.U., Eds.; Springer International Publishing: Cham, Switzerland, 2014; pp. 204-220.

4. Schabus, S.; Scholz, J. Geographic Information Science and technology as key approach to unveil the potential of Industry 4.0: How location and time can support smart manufacturing. In Proceedings of the 12th International Conference on Informatics in Control, Automation and Robotics (ICINCO), Colmar, France, 21-23 July 2015; Volume 2, pp. 463-470.

5. Schabus, S.; Scholz, J.; Skupin, A. Spatial-temporal Patterns of Production Assets in an Indoor Production Environment. In Proceedings of the Workshop “Analysis of Movement Data'14” Workshop at GIScience, Vienna, Austria, 14 February 2014. 
6. Nyström, R.H.; Harjunkoski, I.; Kroll, A. Production optimization for continuously operated processes with optimal operation and scheduling of multiple units. Comput. Chem. Eng. 2006, 30, 392-406. [CrossRef]

7. Li, W.; Goodchild, M.F.; Raskin, R. Towards geospatial semantic search: Exploiting latent semantic relations in geospatial data. Int. J. Digit. Earth 2014, 7, 17-37. [CrossRef]

8. May, G.S.; Spanos, C.J. Fundamentals of Semiconductor Manufacturing and Process Control; John Wiliey \& Sons: Hoboken, NJ, USA, 2006.

9. Geng, H. Semiconductor Manufacturing Handbook; McGraw-Hill: New York, NY, USA, 2005.

10. Gibson, J.J. The Theory of Affordances. In Perceiving, Acting, and Knowing; Shaw, R., Bransford, J., Eds.; Lawrence Erlbaum Associates Inc.: Mahwah, NJ, USA, 1977; pp. 67-82.

11. Gibson, J.J. The Ecological Approach to Visual Perception: Classic Edition; Psychology Press: New York, NY, USA, 2014.

12. Jonietz, D.; Timpf, S. An affordance-based simulation framework for assessing spatial suitability. In Proceedings of the International Conference on Spatial Information Theory, Scarborough, UK, 2-6 September 2013; Springer: Berlin, Germany, 2013; pp. 169-184.

13. Jonietz, D.; Timpf, S. On the relevance of Gibson's affordance concept for geographical information science (GISc). Cognit. Process. 2015, 16, 265-269. [CrossRef] [PubMed]

14. Jonietz, D.; Schuster, W.; Timpf, S. Modelling the suitability of urban networks for pedestrians: An affordance-based framework. In Geographic Information Science at the Heart of Europe; Vandenbroucke, D., Bucher, B., Crompvoets, J., Eds.; Springer: Berlin, Germany, 2013.

15. Davis, J.; Edgar, T.; Porter, J.; Bernaden, J.; Sarli, M. Smart manufacturing, manufacturing intelligence and demand-dynamic performance. Comput. Chem. Eng. 2012, 47, 145-156. [CrossRef]

16. Scholl, A.; Becker, C. State-of-the-art exact and heuristic solution procedures for simple assembly line balancing. Eur. J. Oper. Res. 2006, 168, 666-693. [CrossRef]

17. Osswald, S.; Weiss, A.; Tscheligi, M. Designing wearable devices for the factory: Rapid contextual experience prototyping. In Proceedings of the International Conference on Collaboration Technologies and Systems (CTS), San Diego, CA, USA, 20-24 May 2013; pp. 517-521.

18. Raubal, M. Ontology and epistemology for agent-based wayfinding simulation. Int. J. Geogr. Inf. Sci. 2001, 15, 653-665. [CrossRef]

19. Raubal, M.; Worboys, M. A formal model of the process of wayfinding in built environments. In Spatial Information Theory. Cognitive and Computational Foundations of Geographic Information Science; Springer: Berlin, Germany, 1999; pp. 381-399.

20. Worboys, M. Modeling indoor space. In Proceedings of the 3rd ACM SIGSPATIAL International Workshop on Indoor Spatial Awareness, New York, NY, USA, 1 Novomber 2011.

21. Yang, L.; Worboys, M. Generation of navigation graphs for indoor space. Int. J. Geogr. Inf. Sci. 2015, 29, 1737-1756. [CrossRef]

22. Stoffel, E.P.; Schoder, K.; Ohlbach, H.J. Applying hierarchical graphs to pedestrian in-door navigation. In Proceedings of the 16th ACM SIG Spatial International Conference on Advances in Geographic Information Systems, Irvine, CA, USA, 5-7 Novomber 2008; p. 54.

23. Lorenz, B.; Ohlbach, H.J. A hybrid spatial model for representing indoor environments. In Proceedings of the International Symposium on Web and Wireless Geographical Information Systems, Hong Kong, China, 4-5 December 2006; Springer: Berlin, Germany, 2006; pp. 102-112.

24. Becker, T.; Nagel, C.; Kolbe, T.H. A multilayered space-event model for navigation in indoor spaces. In 3D Geo-Information Sciences; Springer: Berlin, Germany, 2009; pp. 61-77.

25. Stoffel, E.P.; Lorenz, B.; Ohlbach, H.J. Towards a semantic spatial model for pedestrian indoor navigation. In Proceedings of the International Conference on Conceptual Modeling, Auckland, New Zealand, 5-9 November 2007; Springer: Berlin, Germany, 2007; pp. 328-337.

26. Yang, L.; Worboys, M. A navigation ontology for outdoor-indoor space:(work-in-progress). In Proceedings of the 3rd ACM SIGSPATIAL International Workshop on Indoor Spatial Awareness, New York, NY, USA, 1 November 2011; pp. 31-34.

27. Howell, I.; Batcheler, B. Building Information Modeling Two Years Later-Huge Potential, Some Success and Several Limitations. Available online: http://www.laiserin.com/features/bim/newforma_bim.pdf (accessed on 15 June 2017). 
28. Uschold, M.; Gruninger, M. Ontologies: Principles, methods and applications. Knowl. Eng. Rev. 1996, 11, 93-136. [CrossRef]

29. Smith, B. Objects and their environments: From Aristotle to ecological ontology. In Life Motion Socio-Economic Units: Gisdata; Frank, A.U., Ed.; Taylor \& Francis: Abingdon, UK, 2011; Volume 8.

30. Gruber, T.R. A translation approach to portable ontology specification. Knowl. Acquis. 1993, 5, $199-220$. [CrossRef]

31. Davis, E. Representations of Commonsense Knowledge; Morgan Kaufmann: Burlington, MA, USA, 1990.

32. Bishr, Y.; Kuhn, W. Ontology-based modelling of geospatial information. In Proceedings of the 3rd AGILE Conference on Geographic Information Science, Helsinki, Finland, 26-28 May 2000.

33. Grenon, P.; Smith, B. SNAP and SPAN: Towards dynamic spatial ontology. Spat. Cognit. Comput. 2004, 4, 69-104. [CrossRef]

34. Liu, L.; Zlatanova, S. An Approach for Indoor Path Computation among Obstacles that Considers User Dimension. ISPRS Int. J. Geo-Inf. 2015, 4, 2821-2841. [CrossRef]

35. Kostic, N.; Scheider, S. Automated generation of indoor accessibility information for mobility-impaired individuals. In AGILE 2015; Bacao, F., Santos, Y.M., Painho, M., Eds.; Springer: Berlin, Germany, 2015; pp. 235-252.

36. Turner, A.; Penn, A. Encoding natural movement as an agent-based system: An investigation into human pedestrian behaviour in the built environment. Environ. Plan. B Plan. Des. 2002, 29, 473-490. [CrossRef]

37. Kapadia, M.; Singh, S.; Hewlett, W.; Faloutsos, P. Egocentric affordance fields in pedestrian steering. In Proceedings of the 2009 Symposium on Interactive 3D Graphics and Games, Boston, MA, USA, 27 February-1 March 2009.

38. Kim, N.; Joo, J.; Rothrock, L.; Wysk, R.; Son, Y.-J. Human behavioral simulation using affordance-based agent model. In Proceedings of the International Conference on Human-Computer Interaction, Vancouver, BC, Canada, 9-14 July 2011.

39. Richter, K.-F.; Winter, S.; Santosa, S. Hierarchical representations of indoor space. Environ. Plan. B Plan. Des. 2011, 38, 1052-1070. [CrossRef]

40. Hu, Y.; Janowicz, K.; Carral, D.; Scheider, S.; Kuhn, W.; Berg-Cross, G.; Hitzler, P.; Dean, M.; Kolas, D. A geo-ontology design pattern for semantic trajectories. In Proceedings of the International Conference on Spatial Information Theory, Scarborough, UK, 2-6 September 2013; pp. 438-456.

41. Cyganiak, R.; Jentzsch, A. Linking Open Data Cloud Diagram. LOD Community. 2011, Volume 12. Available online: http:/ / lod-cloud (accessed on 15 June 2017).

42. Schabus, S.; Scholz, J. Spatially-Linked Manufacturing Data to support Data Analysis. GI_Forum 2017, 2017, 126-140. [CrossRef]

43. Koffka, K. Principles of Gestalt Psychology; Harcourt Brace: New York, NY, USA, 1935.

44. Jordan, T.; Raubal, M.; Gartrell, B.; Egenhofer, M. An affordance-based model of place in GIS. In Proceedings of the Eight International Symposium on Spatial Data Handling, Vancouver, BC, Canada, 12-15 July 1998; Volume 38, pp. 98-109.

45. Kemke, C. About the Ontology of Actions; Technical Report MCCS-01-328; Computing Research Laboratory, New Mexico State University: Las Cruces, NM, USA, 2001.

46. Leontiev, N. Activity, Consciousness, and Personality; Prentice-Hall: Upper Saddle River, NJ, USA, 1978.

47. Nishi, Y.; Doering, R. Handbook of Semiconductor Manufacturing Technology, 2nd ed.; CRC Press: New York, NY, USA; Basel, Switzerland, 2000.

48. Tsetsos, V.; Anagnostopoulos, C.; Kikiras, P.; Hasiotis, P.; Hadjiefthymiades, S. A human-centered semantic navigation system for indoor environments. In Proceedings of the IEEE International Conference on Pervasive Services (ICPS), Santorini, Greece, 11-14 July 2005.

49. Vanclooster, A.; Ooms, K.; Viaene, P.; Fack, V.; van de Weghe, N.; de Maeyer, P. Evaluating Suitability of the least risk path algorithm to support cognitive wayfinding in indoor space: An empirical study. Appl. Geogr. 2014, 53, 128-140.

(C) 2017 by the authors. Licensee MDPI, Basel, Switzerland. This article is an open access article distributed under the terms and conditions of the Creative Commons Attribution (CC BY) license (http:/ / creativecommons.org/licenses/by/4.0/). 